\title{
Epidemiological and Clinical Data in Low and Intermediate Risk Neuroblastoma: A Single Institution Experience and Survival Outcomes in Jerusalem
}

\author{
Ala H Sbeih ${ }^{1}$, Khadra Salami², Fortunato Morabito ${ }^{3,4}$, Hani Saleh $^{5,6}$ \\ ${ }^{1}$ Al-Quds University School of medicine, Bethlehem, Palestine. ${ }^{2}$ Augusta Victoria Hospital, Ramallah, Palestine. ${ }^{3}$ Biotechnological \\ Research Unit, Provincial Health Authority of Cosenza, Aprigliano (CS), Italy. ${ }^{4}$ Department of Hematology and Bone Marrow \\ Transplant Unit, Augusta Victoria Hospital, Jerusalem, Palestine. ${ }^{5}$ Department of Paediatric Hematology/oncology, Augusta \\ Victoria Hospital, Bethlehem, Palestin. 'Department of Paediatrics at Al-Quds University School of Medicine, East Jerusalem, \\ Palestine.
}

\begin{abstract}
Objective: The aim of this study is to evaluate survival outcomes in neuroblastoma patients referred to Augusta Victoria hospital, Jerusalem between 2009 and 2018. Methods: This is a retrospective study of clinical and epidemiological data evaluating 34 low and intermediate risk neuroblastoma patients treated at a paediatric hematology/oncology center. Information of demographic, treatment modalities, and survival outcome were abstracted from patients medical records. Results: Diagnosing neuroblastoma is challenging due to its wide range of signs and symptoms. In this study, 34 patients were included, with a male to female ratio of 1:26:1.0. The most common location of the tumor is the abdomen followed by the mediastinal region. Forthy one percent of the cases presented with metastasis, mainly to the liver (20\%), bone marrow (15\%), and the lymph nodes (6\%). Overall survival for Low risk neuroblastoma is $100 \%$ while the Intermediate risk neuroblastoma had a $93.1 \%$ chance of survival. Conclusion: The outcome and prognosis of low risk and intermediate risk Neuroblastoma is excellent as it is seen in developed countries. More attention is needed for early detection and early treatment could improve survival.
\end{abstract}

Keywords: Neuroblastoma- risk stratification- survival

Asian Pac J Cancer Care, 5 (3), 139-144

Submission Date: 05/04/2020 Acceptance Date: 07/03/2020

\section{Introduction}

Neuroblastoma has been the second most common solid tumour in children younger than 15 years of age, occupying $8 \%$ of all cancers, with an overall primacy to Leukaemia , brain tumours, lymphomas, respectively. Approximately, 130 new cases of cancer are identified each year per million children in the same age group, with a peak in the first three years of life followed by a decline until the age of 9. On the other hand, a second peak arises in adolescence, taking into consideration that different types of cancers has its own age-distribution pat-tern [1]. However, Survival rates for children have shown improvement during the last decade, the most dramatic improvements occurring among patients with leukemia (48\% 5-year relative survival in 1975-1979 versus $84 \%$ in 2003-2009), non-Hodgkin's lymphoma (47\% versus $85 \%$ ), and others [2].

As the most common malignancy representing al-most $20 \%$ among all neonatal cancers [3], neuroblastoma is derived from neuroepithelial cells developing to form the sympathetic nervous system [4]. It is known for its obscure and preplexed behaviour, some have a regressing turn or maturation process, and some are aggressive despite the different modalities of treatment [5-6].

\section{Materials and Methods}

This is a unicentric retrospective study done at Augusta Victoria hospital (AVH) in Jerusalem, from January

Corresponding Author:

Dr. Hani Saleh

Department of Paediatric Hematology/oncology, Augusta Victoria Hospital, Bethlehem, Palestin.

Department of Paediatrics at Al-Quds University School of Medicine, East Jerusalem, Palestine.

Email: hsaleh@avh.org 
2009 until December 2018, which has been the main referral centre for Palestinian children, considering it is the first and main paediatric hemato-oncology centre in the West Bank and Gaza strip, located in east Jerusalem. It concluded 49 patients from all different districts of the above mentioned areas, who were admitted to our centre to receive treatment, all of which were under the age of 15 . The medical record for each patient was used to obtain the information. All of them had given informed consent for the data.

The diagnosis of neuroblastoma was established based upon the biopsy of the tumour itself with a histopathology examination with immunohistochemical stains, bilateral bone marrow study and urine catecholamines after the review of history, physical examination, laboratory information and radiological studies. Body CT scans were run on all patients to define the primary tumour and the extent of metastasis. Other investigation to complete staging were isotope bone scans and MIBG when available. Tissue samples were obtained through tru cut needle biopsy or an open biopsy, preserved in paraffin block at time of diagnosis along with some fresh tissue sent for biological studies for MYCN gene. Two patients had a case revision histopathology scan after their surgery. bilateral iliac bone punctures were carried on for every patient. MYCN Oncogene amplification measurement were carried out through florescent in situ hybridization method (FISH), at another centre due to lack of facilities, as it is believed to be amplified if more than 10 copies were established per cell. All routine tests were carried out on each patient before starting every cycle of the chemotherapy, comprising hepatic, renal and haematological function, in addition to an echocardiogram whenever needed.

Then, the patients were categorized into stage 1, 2, 3,4 , and 4S, applying the criteria from International Neuroblastoma Staging System (INSS) (Table 1).

All patients in the paediatric Hematology/oncology department at Augusta Victoria Hospital were classified into risks and treated by the international protocol of standard treatment of Children's Oncology Group (COG). One case underwent the europian protocol. Low risk neuroblastoma patients either received no treatment (watchful waiting), partial or complete surgical resection, or chemo-therapy, or a combination of the above mentioned. The current COG protocol (ANBL0531: Response-and Biology-based Therapy for Intermediate-risk Neuroblastoma) was used for intermediate risk group.

High risk group were referred to another centre where autologous bone marrow transplant is available.

The full time period of treatment for low risk patients is from observation or surgery alone or maximum 2 cycles of chemotherapy in case tumor only biopsied or tumor resection less than $50 \%$. For intermediate risk patients ranging from 2 cycles to maximum 8 cycles, follow up period is 5 years, each cycle of chemotherapy is due every 21 days. The outcome results were classified as complete remission, stable disease, refractory disease or tumor recurrence. Complete remission is defined as disease-free status. The stable disease means those who had initial response to treatment then the tumour showed no response to further chemotherapy and is surgically unresectable but no change in size is shown with follow up.

\section{Results}

Through this period, (January 2009- December 2018), 49 patients were diagnosed with neuroblastoma. Of them, 15 cases were transferred to another medical centres either without any intervention, or after diagnosis and staging as those cases were classified as high risk patients, some of them received induction chemotherapy then transferred to a center where autologous bone marrow transplant facility is available.

IBM SPSS Statistics data editor was used to analyze the data.

As such, 34 eligible patients were included in the study with a clear past history of either chemotherapy or radiotherapy, 19 males and 15 female cases.

Median age at diagnosis is 10.29 months, ranging around the antenatal life which was confirmed soon after birth and 8 years old. Of those, the category of $0-12$ months old patients presented the vast majority with of 28 individuals (82\%), 4 patients were between 13-24 months old (12\%), and 2 patients were 24 months and older (6\%).

A male predominance was slight comparing the overall Male: Female ratio of 1.26:1.0.

As for the primary site of the tumour, the most common site is the abdomen (17), then meditational region (11), cervical (2), pelvic (3) and indeterminate bi-adrenal and cervical area (1), with percentages shown in Figure 1.

Of the tumours of an abdominal origin, $11(26 \%)$ originated in the left adrenal gland, $3(14 \%)$ in the right adrenal gland, two (2\%) occurred bilaterally in each adrenal gland, and $1(5 \%)$ was in the retroperitoneal area.

In Table 2, the patients are viewed in order of the time of presentation with the most important characteristics.

At presentation, the most prominent symptoms are abdominal distension ( 8 patients $(23.5 \%)$ ), then fever (6) $(17.6 \%)$, neck swelling (3) $(7 \%)$, respiratory distress (2) $(5.8 \%)$, cough (2) $(5.8 \%)$, periorbital ecchymosis (2) $(5.8 \%)$, and others. Three patients were totally

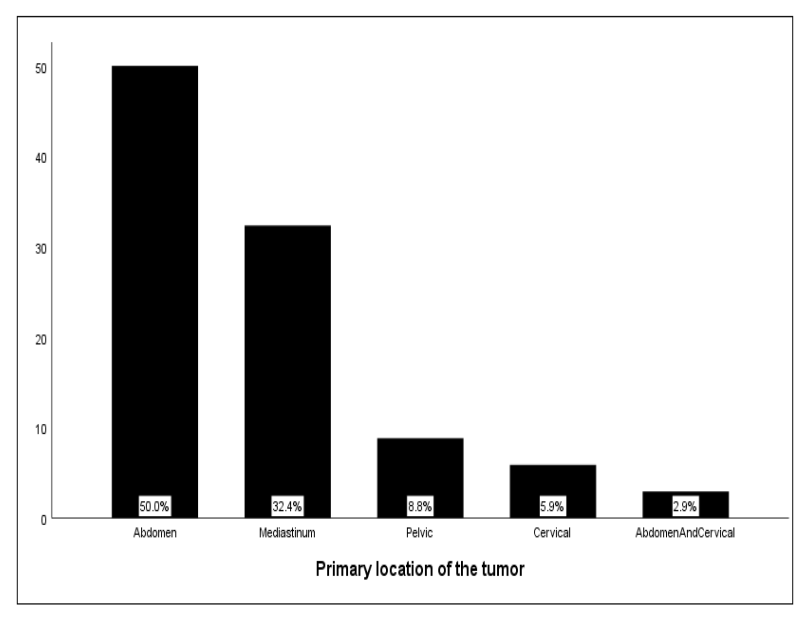

Figure 1. Primary Locations of Neuroblastoma 
Table 1. International Neuroblastoma Staging System [7]

\begin{tabular}{ll}
\hline Stage & Definition \\
\hline 1 & $\begin{array}{l}\text { Localized tumour that is completely resected and may or may not have microscopic residual disease; the representative } \\
\text { ipsilateral lymph nodes are negative (the nodules attached to and removed along with the tumour may be positive). }\end{array}$ \\
2A & $\begin{array}{l}\text { Localized tumour that is not completely resected; representative ipsilateral non-adherent lymph nodes are } \\
\text { microscopically negative for the tumour }\end{array}$ \\
2B & $\begin{array}{l}\text { Localized tumour that may or may not be completely resected with non-adherent ipsilateral lymph nodes that are } \\
\text { positive for the tumour; lymph nodes must be microscopically negative. }\end{array}$ \\
& $\begin{array}{l}\text { Unilateral tumour that extending through the midline, with or without regional lymph node involvement; unilateral } \\
\text { tumour with involvement of regional contralateral lymph nodes; or midline tumour with bilateral extension through } \\
\text { (unresectable) infiltration or lymph node involvement. }\end{array}$ \\
& $\begin{array}{l}\text { Any primary tumour that spreads to distant lymph nodes, bones, bone marrow, the liver, skin, or other organs (except } \\
\text { as defined in stage 4S). }\end{array}$ \\
4S & $\begin{array}{l}\text { Primary localized tumour (as defined for stages 1, 2A or 2B) with limited dissemination to the skin, liver or bone } \\
\text { marrow (limited to children under 1 year of age). }\end{array}$ \\
\hline
\end{tabular}

asymptomatic when they were found to have a mass unrelated to their original com-plaint, or during a regular checkup, in addition to two patients were discovered to have the tumour while going through maternal antenatal care screening.

Other symptoms included dyspnea, constipation, abdominal pain, stridor, dysphagia, elevated blood pressure, vomiting, anemia, lower limb weakness and shoulder pain. 22 out of 34 cases had more than one symptom simultaneously at presentation (64.7\%). 59\% of the cases had no metastasis at time of diagnosis, but of those whom metastasis is present, $20 \%$ are liver metastasis, while $15 \%$ are bone marrow involvement, and $6 \%$ had lymph node metastasis. By age, from the $41 \%$ of overall metastatic cases at presentation, $32 \%$ were between the age of 0- 12 months, then $9 \%$ to $13-24$ months.

To approach the diagnosis of neuroblastoma, $94 \%$ of cases were successful to obtain a biopsy for pathology examination. Then urine catecholamine and biological studies were drawn and sent to an-other hospital due to lack of facility. Of the $94 \%, 47 \%$ were nonfavorable pathology, $18 \%$ were favorable, and $29 \%$ were indeterminate.

Thus, our diagnosis was based upon the histopathology reports obtained after taking a biopsy of the mass.

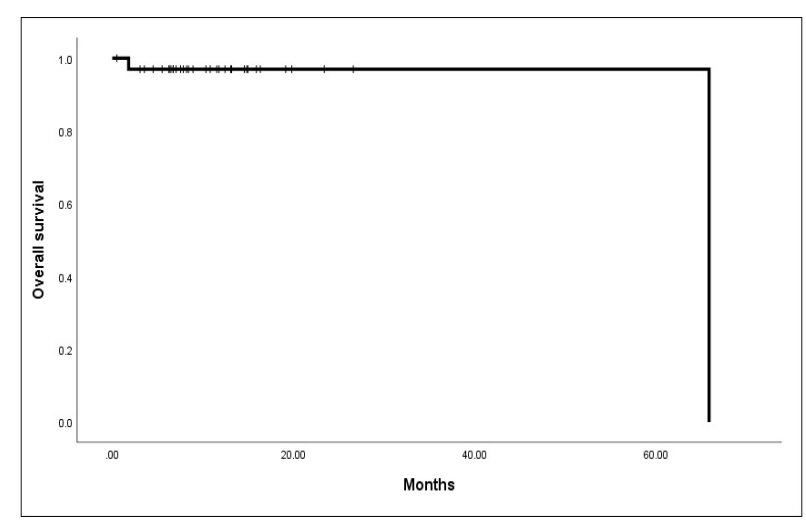

Figure 2. Kaplan Meier Curve for Overall Survival
Otherwise, one case was diagnosed by bone marrow biopsy and one case was started empirically on chemotherapy due to a life-threatening respiratory compromise caused by a mediastinal mass with severe tracheal compression.

Subsequently, MYCN amplification study was done on 24 patients $(71 \%)$, all were nonamplified, the rest are considered as unknown biological feature. Urine for vanillylmandelic acid is tested in 22 cases $(65 \%)$ of all individuals, it outstanded as elevated in 14 patients $(41 \%)$, and not elevated in 8 patients $(24 \%)$. Lack of fund persisted as an obstacle in completing the MYCN amplification test and urine for VMA for the later patients, as those two tests are done at another hospital.

With reference to the INSS system, 44\% were classified as 3, 26\% were stage $4,21 \%$ were stage $4 \mathrm{~s}, 9 \%$ were stage 1 and $0 \%$ are stage 2 . According to the risk stratification, 29 patients were an intermediate risk (85\%), and 5 low risk (15\%) [6].

Looking at the outcome based on the risk stratification, the low risk NB group had a $100 \%$ survival rate. Whilst the intermediate risk NB group, it has a $93.1 \%$ survival rate.

Two mortality cases were encountered, both were females intermediate risk neuroblastomas; the first one is a neonatal stage 4 with massive hepatomegaly and $\mathrm{BM}$ involvement $>10 \%$, it experienced progression on treatment and was transferred to another center and later died despite aggressive chemotherapy and autologous stem cell transplant. Another patient, the second case (age $>1 \mathrm{y}$, stage III mediastinal FH) underwent remission after surgery and chemotherapy, followed by cervical lymph node recurrence of the tumour after two years. Kaplan Meier's curves for survival was used to demonstrate the cases in Figure 2. General acute complications among the management were acute infections as yeast sepsis or bacterial sepsis. The long term complications observed due to tumor effect were Horner syndrome, scoliosis and poor growth and permanent lower limb paralysis.

\section{Discussion}

Neuroblastoma is the most common extra cranial solid tumour in children, it accounts approximately for 
Table 2. Neuroblastoma Patients Characteristics with Disease Out come from 2009-2018

\begin{tabular}{|c|c|c|c|c|c|c|c|c|}
\hline Patient's Number & Age & Sex & Location & $\begin{array}{c}\text { MYCN } \\
\text { Amplification }\end{array}$ & Stage & Risk & Treatment & Outcome \\
\hline 1 & $10 \mathrm{mos}$ & $\mathrm{F}$ & Mediastinum & non-amplified & 3 & IR & COG IR & Stable disease \\
\hline 2 & $6 \mathrm{mos}$ & M & Sacrum & non-amplified & 3 & IR & COG IR & Complete remission \\
\hline 3 & $4 \mathrm{yrs}$ & $\mathrm{F}$ & Mediastinum & non-amplified & 3 & IR & $\begin{array}{l}\text { Europian protocol } \\
\text { for IR NB }\end{array}$ & Recurrence, Died. \\
\hline 4 & $4 \operatorname{mos}$ & $\mathrm{F}$ & Left Adrenal & Unknown & $4 \mathrm{~s}$ & IR & COG IR & Complete remission \\
\hline 5 & $7 \mathrm{mos}$ & $\mathrm{F}$ & Mediastinum & Unknown & 3 & IR & COG IR & Complete remission \\
\hline 6 & $10 \mathrm{mos}$ & $\mathrm{F}$ & Cervical & non-amplified & 3 & IR & COG IR & Complete remission \\
\hline 7 & $7 \mathrm{yrs}$ & M & Left Adrenal & non-amplified & 1 & LR & Watchful waiting & Complete remission \\
\hline 8 & $7 \mathrm{mos}$ & M & Mediastinum & non-amplified & 3 & IR & COG IR & Complete remission \\
\hline 9 & $8 \mathrm{mos}$ & M & Left adrenal & non-amplified & 4 & IR & COG IR & Complete remission \\
\hline 10 & Antenatally & M & Left adrenal & Unknown & 1 & LR & Watchful waiting & Complete remission \\
\hline 11 & $1 \mathrm{yr}$ & M & Right adrenal & non-amplified & 3 & IR & COG IR & Complete remission \\
\hline 12 & $1 \mathrm{mos}$ & $\mathrm{F}$ & Liver & Unknown & $4 \mathrm{~s}$ & IR & COG IR & Complete remission \\
\hline 13 & $1 \mathrm{mos}$ & $\mathrm{F}$ & Left adrenal & Unknown & 3 & LR & Watchful waiting & Complete remission \\
\hline 14 & $1 \mathrm{mos}$ & $\mathrm{F}$ & Right adrenal & Non-amplified & 4 & IR & COG IR & Refractory, Died. \\
\hline 15 & $3 \mathrm{mos}$ & M & Cervical & Unknown & 4 & IR & COG IR & Complete remission \\
\hline 16 & $2 \mathrm{mos}$ & M & Adrenal; Bilateral & non-amplified & 4 & LR & Watchful waiting & Complete remission \\
\hline 17 & $3 \mathrm{mos}$ & $\mathrm{F}$ & Left adrenal & non-amp & $4 \mathrm{~s}$ & IR & COG IR & Complete remission \\
\hline 18 & $1.2 \mathrm{yrs}$ & M & Left adrenal & non-amplified & 4 & IR & COG IR & Complete remission \\
\hline 19 & Antenatally & M & Right adrenal & non-amplified & $4 \mathrm{~s}$ & IR & COG IR & Complete remission \\
\hline 20 & $7 \mathrm{mos}$ & $\mathrm{F}$ & Mediastinum & non-amplified & 3 & IR & COG IR & Complete remission \\
\hline 21 & $1 \mathrm{yr}$ & $\mathrm{F}$ & Left adrenal & Unknown & 1 & LR & Excisional Biobsy & Complete remission \\
\hline 22 & $6 \mathrm{mos}$ & $\mathrm{F}$ & Mediastinum & Unknown & 3 & IR & COG IR & Stable disease \\
\hline 23 & $2 \mathrm{mos}$ & $\mathrm{F}$ & Abdomen & non-amplified & $4 \mathrm{~s}$ & IR & COG IR & Stable disease \\
\hline 24 & $2 \mathrm{mos}$ & M & Adrenal; Bilateral & non-amplified & $4 \mathrm{~s}$ & IR & COG IR & Complete remission \\
\hline 25 & $5 \mathrm{mos}$ & F & Left adrenal & non-amplified & $4 \mathrm{~s}$ & IR & COG IR & Stable disease \\
\hline 26 & $1 \mathrm{yr}$ & M & Pelvic & Unknown & 3 & IR & COG IR & Complete remission \\
\hline 27 & $6 \mathrm{mos}$ & $\mathrm{F}$ & Left adrenal & non-amplified & 4 & IR & COG IR & Complete remission \\
\hline 28 & $1 \mathrm{yr}$ & M & Mediastinum & non-amplified & 4 & IR & COG IR & Stable disease \\
\hline 29 & $7 \mathrm{mos}$ & M & Mediastinum & non-amplified & 4 & IR & COG IR & Stable disease \\
\hline 30 & $2 \mathrm{mos}$ & M & Mediastinum & Unknown & 3 & IR & COG IR & Complete remission \\
\hline 31 & $1.5 \mathrm{yrs}$ & M & Mediastinum & non-amplified & 3 & IR & COG IR & Stable disease \\
\hline 32 & $6 \operatorname{mos}$ & M & Pelvic & non-amplified & 3 & IR & COG IR & Complete remission \\
\hline 33 & $1.2 \mathrm{yrs}$ & M & $\begin{array}{l}\text { Abdomen; retroperitoneal } \\
\text { space }\end{array}$ & non-amplified & 4 & IR & COG IR & Stable disease \\
\hline 34 & $9 \mathrm{mos}$ & M & Mediastinum & non-amplified & 3 & IR & COG IR & Complete remission \\
\hline
\end{tabular}

i.e, Mos, months; Yrs, years; F, female; M, male; IR, intermediate risk; LR, low risk; COG IR, Children oncology group protocol for Intermediate risk neuroblastoma.

$8 \%$ of all childhood cancers. Detecting and diagnosing neuroblastoma is challenging mostly due to its variable and nonspecific signs and symptoms. Risk stratification is very important in determining the treatment strategy and prognosis, it depends on the patient's age and stage at diagnosis, MYCN status as a biological factor, histopathological classification plus the DNA pleiody [1-8].

MYCN amplification has shown relation to determining the aggressiveness of neuroblastoma as it is in $16-25 \%$ of cases [9-10], in addition to Anaplastic lymphoma kinase gene (ALK) which is a major predisposition to familial neuroblastoma [11]. Happening that MYCN amplification and ALK gene mutation might get-together in the same case produces a poor output suggesting a cooperation between the two [12].

Patients are classified into low, intermediate, and high risk groups based on the age, stage, MYCN status and histology, while the prognosis is based on risk type, response to treatment, and the time interval between diagnosis and recurrence (relapse cases) [13].

Patients of all ages with stage 1,2 , or $4 \mathrm{~S}$ disease without MYCN amplification have an excellent prognosis with a 5 -year survival rate of over $95 \%$. Patients with stage 3 , as well as infants with stage 4 neuroblastoma without MYCN amplification have a survival rate of approximately $75 \%$. Children ( $>1$ year) with stage 4 neuroblastoma (regardless of MYCN status), MYCN amplified stage 2 and 3 disease, as well as infants less than 1 year with metastatic, MYCN amplified disease has 
around $30 \%$ chance of recovery [14].

Here in our study, the most prevalent symptom was abdominal distention, taking into consideration that many patients presented with more than one com-plaint. Other than this, the symptoms occurred related to the location of the tumour, thus, some cases had an abdominal distention alongside with constipation or vomiting. On the other hand, patients presented with cervical or mediastineal mass had respiratory symptoms, explaining cough, stridor, or, with more complexity, a respiratory tract infection. Other symptoms were fever, periorbital ecchymosis, and anemia suggested metastasis.

A small percentage were asymptomatic and were dis-covered by doing a physical exam or a radiological study for yet another reason not related to the diagnosis of neuroblastoma. Ages by which the disease was diagnosed at had a median of 21.09 months for all risk types, a study by London, W. B. et al showed the that median age at diagnosis is 18 months [15], this can be explained by our small sample size. The primary site of the tumor were, in order, the abdomen, mediastinum, cervical , pelvic regions.

As for metastasis of LR and IR group, the most common site is the liver, then the bone marrow and lymph nodes. In our study, $85 \%$ of our cases were of an intermediate risk and $15 \%$ were low risk. High risk patients were transferred to another centers due to lack of facilities. Most of them were under the age of one year. Looking at the outcome results, low risk neuroblastoma patients had a 100\% 5-year survival, while the intermediate risk had a percentage of $93 \%$. as more than one study approved that increasing age is associated with worse outcome results [16-17], Pinto et al clarified in his research the low risk neuroblastoma patients more than $95 \%$ chance of survival at 5-year follow up, and intermediate risk neuroblastoma between 90 and $95 \%$ at the same time interval [18], our better survival results in IR group is mostly is because that majority of the patients are less than 1 year (82\%).

Survival post-relapse is dismal, A study by Garaventa et al [19], showed that the Survival of children with recurrent neuroblastoma is very poor. Treatment complications were bacterial and fungal infections and sepsis. Long term sequelae which are related to the tumor effect are the most dramatic as in our patients that included Horner syndrome, scoliosis, and permanent lower limb paralysis.

Looking into limitations, AVH has been a referral centre since 2008 from the West Bank and Gaza Strip for Palestinian children, so the load on the hospital had become overstrained at some points. In 2012, two centres opened in the west bank, so cases are shared between AVH and them. Lack of enough instrumentation has always been a chal-lenge till this day.

Funding and coverage of some important studies including biological study, and imaging, mainly MIBG, is a major challenge for optimal staging and management of neuroblastoma cases in Palestine. Finally, number of cases of neuroblastoma are ex-pected to increase as the populations grows in the previously mentioned districts, but the diagnosis of Neuroblastoma itself is still a stump due to the tumour's perplexed presentation and behaviour and not enough tools to diagnose it.

In conclusion, the outcome and prognosis of low risk and inter-mediate risk Neuroblastoma is excellent as in developed countries, more attention and medical awareness for early detection and treatment of IR neuroblastoma to minimize severe long term tu-mour morbidities in these groups is crucial.

\section{Funding}

This study did not receive any funding.

\section{Conflict of interests}

The authors have no conflicts to declare.

\section{Abbreviations}

NB, Neuroblastoma; LR, Low risk; IR, Intermediate risk; HR, High risk; FISH, florescent in situ hybridization method; VMA, Vanillylmandelic acid.

\section{References}

1. Davidoff AM. Pediatric oncology. Seminars in Pediatric Surgery. 2010 08;19(3):225-233. https://doi.org/10.1053/j. sempedsurg.2010.03.007

2. Ward E, DeSantis C, Robbins A, Kohler B, Jemal A. Childhood and adolescent cancer statistics, 2014. CA: A Cancer Journal for Clinicians. 201401 31;64(2):83-103. https://doi. org/10.3322/caac.21219

3. Fisher JP, Tweddle DA. Neonatal neuroblastoma. Seminars in Fetal and Neonatal Medicine. 2012 08;17(4):207-215. https://doi.org/10.1016/j.siny.2012.05.002

4. Cheung NV, Dyer MA. Neuroblastoma: developmental biology, cancer genomics and immunotherapy. Nature Reviews Cancer. 201305 24;13(6):397-411. https://doi. org $/ 10.1038 / \mathrm{nrc3526}$

5. Nakazawa A, Haga C, Ohira M, Okita H, Kamijo T, Nakagawara A. Correlation between the International Neuroblastoma Pathology Classification and genomic signature in neuroblastoma. Cancer Science. 201504 22;106(6):766-771. https://doi.org/10.1111/cas.12665

6. Cohn SL, Pearson AD, London WB, Monclair T, Ambros PF, Brodeur GM, Faldum A, Hero B, Iehara T, Machin D, Mosseri V, Simon T, Garaventa A, Castel V, Matthay KK. The International Neuroblastoma Risk Group (INRG) Classification System: An INRG Task Force Report. Journal of Clinical Oncology. 200901 10;27(2):289-297. https://doi. org/10.1200/jco.2008.16.6785

7. Brodeur GM, Pritchard J, Berthold F, Carlsen NL, Castel V, Castelberry RP, De Bernardi B, Evans AE, Favrot M, Hedborg F. Revisions of the international criteria for neuroblastoma diagnosis, staging, and response to treatment.. Journal of Clinical Oncology. 1993 08;11(8):1466-1477. https://doi.org/10.1200/jco.1993.11.8.1466

8. Look AT, Hayes FA, Shuster JJ, Douglass EC, Castleberry RP, Bowman LC, Smith EI, Brodeur GM. Clinical relevance of tumor cell ploidy and N-myc gene amplification in childhood neuroblastoma: a Pediatric Oncology Group study.. Journal of Clinical Oncology. 1991 04;9(4):581-591. https://doi. org/10.1200/jco.1991.9.4.581

9. Seeger RC, Brodeur GM, Sather H, Dalton A, Siegel SE, Wong KY, Hammond D. Association of Multiple Copies of the N-mycOncogene with Rapid Progression of Neuroblastomas. New England Journal of Medicine. 1985 Oct 31;313(18):1111-1116. https://doi.org/10.1056/ 
nejm198510313131802

10. Bagatell R, Beck-Popovic M, London WB, Zhang Y, Pearson AD, Matthay KK, Monclair T, Ambros PF, Cohn SL. Significance of MYCN Amplification in International Neuroblastoma Staging System Stage 1 and 2 Neuroblastoma: A Report From the International Neuroblastoma Risk Group Database. Journal of Clinical Oncology. 200901 20;27(3):365-370. https://doi.org/10.1200/jco.2008.17.9184

11. Mossé YP, Laudenslager M, Longo L, Cole KA, Wood A, Attiyeh EF, Laquaglia MJ, Sennett R, Lynch JE, Perri P, Laureys G, Speleman F, Kim C, Hou C, Hakonarson H, Torkamani A, Schork NJ, Brodeur GM, Tonini GP, Rappaport E, Devoto M, Maris JM. Identification of ALK as a major familial neuroblastoma predisposition gene. Nature. 200808 24;455(7215):930-935. https://doi.org/10.1038/ nature 07261

12. De Brouwer S, De Preter K, Kumps C, Zabrocki P, Porcu M, Westerhout EM, Lakeman A, Vandesompele J, Hoebeeck J, Van Maerken T, De Paepe A, Laureys G, Schulte JH, Schramm A, Van Den Broecke C, Vermeulen J, Van Roy N, Beiske K, Renard M, Noguera R, Delattre O, JanoueixLerosey I, Kogner P, Martinsson T, Nakagawara A, Ohira M, Caron H, Eggert A, Cools J, Versteeg R, Speleman F. Meta-analysis of Neuroblastomas Reveals a Skewed ALK Mutation Spectrum in Tumors with MYCN Amplification. Clinical Cancer Research. 201008 18;16(17):4353-4362. https://doi.org/10.1158/1078-0432.ccr-09-2660

13. Jiang M, Stanke J, Lahti J. The Connections Between Neural Crest Development and Neuroblastoma. Cancer and Development. 2011;:77-127. https://doi.org/10.1016/ b978-0-12-380916-2.00004-8

14. Schell, Matthias, and Christophe Bergeron. "Neuroblastoma." Orpha.net, Oct. 2003, www.orpha.net/data/patho/GB/ukneuroblastoma.pdf..

15. London W, Castleberry R, Matthay K, Look A, Seeger R, Shimada H, Thorner P, Brodeur G, Maris J, Reynolds C, Cohn S. Evidence for an Age Cutoff Greater Than 365 Days for Neuroblastoma Risk Group Stratification in the Children's Oncology Group. Journal of Clinical Oncology. 200509 20;23(27):6459-6465. https://doi.org/10.1200/ jco.2005.05.571

16. Taggart DR, London WB, Schmidt ML, DuBois SG, Monclair TF, Nakagawara A, De Bernardi B, Ambros PF, Pearson AD, Cohn SL, Matthay KK. Prognostic Value of the Stage 4S Metastatic Pattern and Tumor Biology in Patients With Metastatic Neuroblastoma Diagnosed Between Birth and 18 Months of Age. Journal of Clinical Oncology. 2011 Nov 20;29(33):4358-4364. https://doi.org/10.1200/ jco.2011.35.9570

17. Schmidt ML, Lal A, Seeger RC, Maris JM, Shimada H, O'Leary M, Gerbing RB, Matthay KK. Favorable Prognosis for Patients 12 to 18 Months of Age With Stage 4 Nonamplified MYCN Neuroblastoma: A Children's Cancer Group Study. Journal of Clinical Oncology. 200509 20;23(27):6474-6480. https://doi.org/10.1200/ jco.2005.05.183

18. Pinto NR, Applebaum MA, Volchenboum SL, Matthay KK, London WB, Ambros PF, Nakagawara A, Berthold F, Schleiermacher G, Park JR, Valteau-Couanet D, Pearson AD, Cohn SL. Advances in Risk Classification and Treatment Strategies for Neuroblastoma. Journal of Clinical Oncology. 201509 20;33(27):3008-3017. https://doi.org/10.1200/ jco.2014.59.4648

19. Garaventa A, Parodi S, De Bernardi B, Dau D, Manzitti C, Conte M, Casale F, Viscardi E, Bianchi M, D'Angelo P, Zanazzo GA, Luksch R, Favre C, Tamburini A, Haupt R.
Outcome of children with neuroblastoma after progression or relapse. A retrospective study of the Italian neuroblastoma registry. European Journal of Cancer. 2009 Nov;45(16):28352842. https://doi.org/10.1016/j.ejca.2009.06.010

This work is licensed under a Creative Commons AttributionNon Commercial 4.0 International License. 